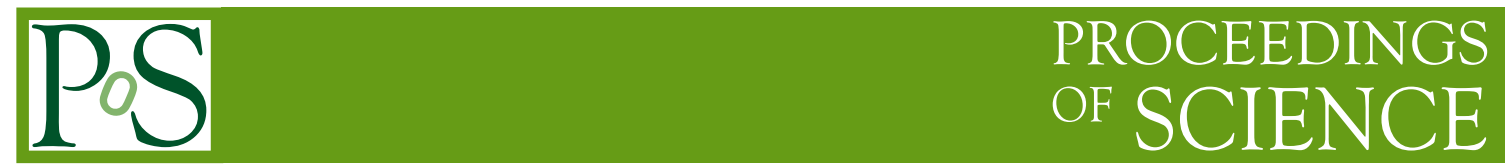

\title{
Neutrinoless Double Beta Decay
}

\author{
Fabio Bellini* \\ Dip. di Fisica dell'Università di Roma Sapienza and Sez. INFN di Roma, \\ Roma I-00185, Italy \\ E-mail: fabio.bellini@romal.infn.it
}

\begin{abstract}
The study of neutrino properties is one of the fundamental challenges in Particle Physics nowadays. Fifty years of neutrino investigations established that neutrinos are massive particles but the absolute scale has not yet been measured. Moreover the true nature of the neutrino mass is still unknown. Is the neutrino its own antiparticle, as proposed by Majorana in 1937? The only way to probe the neutrino nature and provide a measurement of its mass is through the observation of Neutrinoless Double Beta Decay, a very rare, spontaneous nuclear transition which emits two electrons and no neutrinos. An overview of present status and future perpectives of $0 v \beta \beta$ experimental searches is given.
\end{abstract}

Flavor Physics and CP Violation - FPCP 2010

May 25-29, 2010

Turin, Italy

* Speaker. 


\section{Introduction}

The discovery of neutrino oscillation in solar, atmospheric and reactor experiments $[1,2,3,4$, 5] has renewed the interest of the Neutrinoless Double Beta Decay $(0 v \beta \beta)$ as a fundamental and unique probe for the neutrino nature, that could also investigate the neutrino absolute mass scale through the measurement of the effective Majorana mass. Neutrino oscillation experiments have provided indirect evidence for finite neutrino masses. They are sensitive to the values of the squared mass differences between the neutrino species measured, leaving room for two possible hierarchical mass arrangements of neutrino masses (direct and inverted), besides the quasi-degenerate option.

The importance of neutrino masses is also related to the question of the neutrino nature. Neutrinos, in fact, are the only fermions for which the Majorana formulation [6] is possible, assuming a violation of the Lepton Number. This description is indistinguishable from the Dirac one in the limit of vanishing masses. Until the discovery of the massive nature of neutrinos little attention was therefore dedicated to the issue of Majorana neutrinos. The situation has changed after 1998 and there is a common consensus that Majorana description is indeed the best description for the physical neutrinos.

From an experimental point of view, Neutrinoless Double Beta Decay is the only accessible process able to test the neutrino nature. Moreover it is a powerful tool to investigate possible extensions of the Standard Model since it violates the lepton number by two units and an observation would imply new Physics beyond the Standard Model.

\section{Neutrinoless Double Beta Decay}

The Double Beta Decay is a rare spontaneous nuclear transition, proposed by M.GoeppertMayer in 1935 [7], in which a nucleus (A,Z) decays to a member (A,Z+2) of the same isobaric multiplet with the simultaneous emission of two electrons. Both the parent and the daughter nuclei should be more bound than the intermediate one, in order to avoid the occurrence of the equivalent sequence of two single beta decays. Such a condition, due to the pairing term, is fulfilled in nature for 35 naturally occurring even-even nuclei. The decay can proceed both to the ground state or to the first excited states of the daughter nucleus. Being a second-order process of the weak interaction, it has a very low probability which leads to extraordinary long lifetimes for the candidate nuclides. Double beta transitions accompanied by positron emission or electron capture are also possible but they are usually characterized by lower transition energies and poorer experimental sensitivities and therefore they will not discussed here.

Double beta decay modes could be further classified in $2 v \beta \beta$ and $0 v \beta \beta$.

The $2 v \beta \beta$ mode ${ }_{Z}^{A} X \rightarrow{ }_{Z+2}^{A} X+2 e^{-}+2 \bar{v}$ is allowed by the Standard Model of electro-weak interactions and satisfies the lepton number conservation.

The $0 v \beta \beta$ mode ${ }_{Z}^{A} X \rightarrow{ }_{Z+2}^{A} X+2 e^{-}$, on the other hand, violates the lepton number by two units and can occur if neutrinos are their own antiparticles. It was introduced for the first time by W.H. Furry [8] in 1939 and, in the light virtual Majorana neutrino exchange scheme, its half-life is expressed as

$$
\left[T_{1 / 2}^{0 v}\right]^{-1}=G^{0 v}\left|M^{0 v}\right|^{2}\left|m_{\beta \beta}\right|^{2}
$$


where $G^{0 v}$ is the accurately calculable phase space integral, $\left|M^{0 v}\right|^{2}$ is the nuclear matrix element and $m_{\beta \beta}$, the effective Majorana mass, is a linear combination of the neutrino masses:

$$
m_{\beta \beta} \equiv \sum_{k=1}^{3}\left|U_{e k}\right|^{2} m_{k} e^{i \phi_{k}}
$$

In equation 2.2, $U_{e k}$ are the elements of the neutrino mixing matrix, $m_{k}$ are the neutrino masses and $\phi_{k}$ are the Majorana phases. The presence of $\phi_{k}$ phaces in the $m_{\beta \beta}$ expression could lead to cancellations. Those are complete for a Dirac neutrino since it is equivalent to two degenerate Majorana neutrinos with opposite CP phases.

Given the actual knowledge of neutrino masses and mixing parameters, two possible orderings are possible: direct and inverted $\left(m_{\beta \beta} \sim 20-50 \mathrm{meV}\right)$. In the case that next $0 v \beta \beta$ experiments would not observe any decay the inverse ordering could be excluded thus fixing the problem of the neutrino absolute mass scale $[9,10] . m_{\beta \beta}$ is actually the only $0 v \beta \beta$ measurable parameter containing direct information on the neutrino mass scale. Its extraction from the experimental results on $0 v \beta \beta$ half-lifetime requires, however, a precise knowledge of the transition Nuclear Matrix Elements $\mathrm{M}^{0 v}$. Different evaluations, available in literature, exhibit considerable disagreement among themselves, leading to large uncertainty ranges for $m_{\beta \beta}$.

\section{Double Beta Decay experimental approaches}

From an experimental point of view, all the available information in $\beta \beta$ decay is carried out by the two electrons and the daughter nucleus.

The main signature of the $0 v \beta \beta$ decay is a peak in the sum energy spectrum of the electrons at the $\mathrm{Q}$ value of the reaction while in $2 v \beta \beta$ mode the spectrum is a continuum between 0 and $\mathrm{Q}$ with a maximum around one third of $\mathrm{Q}$.

Additional signatures are: the single electron energy and angular distribution, identification of the daughter nucleus.

The performance of the different $0 v \beta \beta$ experiments is usually expressed in terms of an experimental sensitivity or factor of merit $\left(F_{0 v}\right)$, defined as the process half-life corresponding to the maximum signal $\mathrm{n}_{B}$ that could be hidden by the background fluctuations at a given statistical C.L. At $1 \sigma$ level $\left(\mathrm{n}_{B}=\sqrt{B T M \Delta}\right)$, one obtains:

$$
F_{0 v}=\tau_{1 / 2}^{\text {Back.Fluct. }}=\ln 2 N_{\beta \beta} \varepsilon \frac{T}{n_{B}}=\ln 2 \times \frac{x \eta \varepsilon N_{A}}{A} \sqrt{\frac{M T}{B \Delta}}(68 \% C L)
$$

where $\mathrm{B}$ is the background level per unit mass and energy, $\mathrm{M}$ is the detector mass, $\mathrm{T}$ is the measure time, $\Delta$ is the FWHM energy resolution, $\mathrm{N}_{\beta \beta}$ is the number of $\beta \beta$ decaying nuclei under observation, $\eta$ their isotopic abundance, $\mathrm{N}_{A}$ the Avogadro number, A the compound molecular mass, $x$ the number of $\beta \beta$ atoms per molecule, and $\varepsilon$ the detection efficiency.

Despite its simplicity, equation 3.1 has the advantage of emphasizing the role of the essential experimental parameters: mass, measuring time, isotopic abundance, background level and detection efficiency.

The desirable features for $0 v \beta \beta$ detectors are therefore: 
- Low background, which requires underground detector operation to shield cosmic rays, very radio-pure materials (natural radioactivity decays have typical lifetimes of the order $10^{9 / 10} \mathrm{y}$ versus lifetimes longer than $10^{25} \mathrm{y}$ for $0 v \beta \beta$ decay) and well-designed passive and/or active shielding against local environmental radioactivity.

- Large source, in order to monitor many candidates nuclei.

- High energy resolution, since a peak must be identified over an almost flat background.

- Event reconstruction, useful to reject background and provide additional kinematical information on emitted electrons.

- A reliable and easy to operate detector technology requiring a minimum level of maintenance (long underground running times).

All those features could be not optimized simultaneously in a single detector. Different experimental approaches are exploited and different searches are usually classified in homogeneous technique when the $\beta \beta$ source is embedded in the detector and inhomogeneous technique when the observed electrons originate in an external source. Calorimetric detectors are usually preferred for future experiments since they have produced so far the best results.

\subsection{Present Experimental situation}

Experimental evidence for $2 v \beta \beta$ decays and upper limit for $0 v \beta \beta$ half-lifes decays are summarized in Tab. 1. The best results are still maintained by the use of isotopically enriched High Purity Ge diodes for the experimental investigation of ${ }^{76} \mathrm{Ge}$ (Heidelberg-Moscow (HM) [11] and IGEX[12]) but two other experiments have recently reached comparable sensitivities: NEMO3 $[13,14]$ at LSM and CUORICINO at LNGS [15].

The HM experiment operated five Ge diodes $\left(86 \%{ }^{76} \mathrm{Ge}\right.$ enriched) at LNGS with high energy resolution ( $\sim 4 \mathrm{keV}$ FWHM) and extremely low background ( 0.17 counts/keV/ky/y). In $2001 \mathrm{a}$ subset (KHDK) of the HM collaboration claimed (and recently confirmed [16]) evidence for a $0 v \beta \beta$ signal with $T_{1 / 2}^{0 v}=2.23_{-0.31}^{+0.44} \times 10^{25} \mathrm{y}$. The result, based on a re-analysis of the HDM data, has raised some criticism but cannot be dismissed out of hand.

NEMO3 is an inhomogeneous detector in which the electrons emitted by the sources cross a magnetized tracking volume instrumented with Geiger cells and deliver their energy to a plastic scintillating calorimeter. The big advantage of the NEMO3 technique is the possibility to study many candidates simultaneously and to access single electron informations which allow $2 v \beta \beta$ decays to be reconstructed with no other background. On the other hand, the poor energy resolution $(\sim 12 \%$ FWHM) and the limited amount of $0 v \beta \beta$ mass source (due to unavoidable source-foil structure) limits the $0 v \beta \beta$ sensitvity. NEMO3 will continue data taking until the end of 2010.

CUORICINO [15] is an array of $62 \mathrm{TeO}_{2}$ bolometers with a total active mass of $40.7 \mathrm{~kg}$, that corresponds to a mass of ${ }^{130} \mathrm{Te}$ of $\sim 11 \mathrm{~kg}$. Bolometers are sensitive calorimeters, operated at a few milli-Kelvin, that measure the rise in temperature due to the energy deposited by particle interactions. CUORICINO took data in the period 2003-2008. A very low background, comparable with the HM, was achieved together with an average resolution of $\sim 7 \mathrm{keV}$ FWHM. Unfortunately 
it can't disprove the HM claim due to the large uncertainties in the Nuclear Matrix element calculations. The only certain way to confirm or refute it is with additional sensitive experiments.

Table 1: Best reported results on $\beta \beta$ processes. $0 v \beta \beta$ limits are at $90 \% \mathrm{CL}$ while $2 v \beta \beta$ results are averaged values taken, except when explicitely noted, from [17]. Future developments of the technique are shown in the last columns together with their expected location and isotope mass.

\begin{tabular}{|c|c|c|c|c|c|}
\hline Isotope & $\begin{array}{c}\mathrm{T}_{1 / 2}^{2 v} \\
\left(10^{19} \mathrm{y}\right)\end{array}$ & $\begin{array}{c}\mathrm{T}_{1 / 2}^{0 v} \\
\left(10^{24} \mathrm{y}\right)\end{array}$ & $\begin{array}{l}\text { Future } \\
\text { Experiment }\end{array}$ & $\begin{array}{c}\text { Mass } \\
(\mathrm{kg})\end{array}$ & Lab \\
\hline$\overline{{ }^{48} \mathrm{Ca}}$ & $\left(4.4_{-0.5}^{+0.6}\right)$ & $>0.0014[18]$ & CANDLES & & OTO \\
\hline${ }^{76} \mathrm{Ge}$ & $(150 \pm 10)$ & $\begin{array}{c}>19[11] \\
22.3_{-3.1}^{+4.4}[16]\end{array}$ & GERDA & $18-40$ & LNGS \\
\hline${ }^{82} \mathrm{Se}$ & $(9.2 \pm 0.7)$ & $>0.36[14]$ & SuperNEMO & 100 & LSM \\
\hline${ }^{96} \mathrm{Zr}$ & $(2.3 \pm 0.2)$ & $>0.0092[14]$ & & & \\
\hline${ }^{100} \mathrm{Mo}$ & $(0.71 \pm 0.04)$ & $>1.1[14]$ & MOON & & OTO \\
\hline${ }^{116} \mathrm{Cd}$ & $(2.8 \pm 0.2)$ & $>0.17[19]$ & & & \\
\hline${ }^{130} \mathrm{Te}$ & $(68 \pm 12)$ & $>2.94$ & CUORE & 204 & LNGS \\
\hline \multirow[t]{2}{*}{${ }^{136} \mathrm{Xe}$} & $>81[20]$ & $>0.12[21]$ & EXO & 160 & WIPP \\
\hline & & & KAMLAND & 200 & KAMIOKA \\
\hline${ }^{150} \mathrm{Nd}$ & $(0.82 \pm 0.09)$ & $>0.0036[22]$ & $\mathrm{SNO}+$ & 56 & SNOLAB \\
\hline
\end{tabular}

\subsection{Future strategies and forthcoming experiments}

The goal of next generation experiments is to reach a sensitivity able to to explore the inverted hierarchy (IH) of neutrino masses $\left(m_{\beta \beta} \sim 20-50 \mathrm{meV}\right)$. From an experimental point of view this corresponds to active masses of the order of 1 ton with background levels of the order of 1 count $/ \mathrm{keV} / \mathrm{ton} / \mathrm{y}$. This requires control of contamination very close or below the current diagnostic technology. Phased programs have been therefore proposed. Second generation experiments are all characterized by hundred $\mathrm{kg}$ detectors and 1-10 counts/kev/ton/y background rates. Their goal is to select the best technology for approaching the IH region.

A list of some of the forthcoming $0 v \beta \beta$ projects is given in Tab. 1.

Among calorimeter detectors three main categories can be identified: high energy resolution without tracking capability (GERDA, CUORE), modest energy resolution with event topology reconstruction (EXO) and modest energy resolution with large mass (SNO+, KAMLAND).

GERDA [23], evolved from the Heidelberg-Moscow experiment, is an array of enriched Ge diodes operated in a LAr bath [24] for background suppression. The main advantage of the GERDA experiment consists in the well understood performance of germanium detectors besides an excellent resolution of $\sim 0.16 \%$ FWHM that guarantees a negligible contamination of the $0 v \beta \beta$ signal from $2 v \beta \beta$ decays. An important issue for background suppression is granularity thanks to the rejection of simultaneous events in different detectors that could not be ascribed to $0 v \beta \beta$ decay. Another tool to improve the sensitivity is provided by the pulse shape discrimination, already used in the Heidelberg-Moscow experiment with remarkable results. The GERDA setup construction is 
presently being completed in Gran Sasso. $18 \mathrm{~kg}$ of Germanium detectors enriched in ${ }^{76} \mathrm{Ge}$ are used in the first phase of the experiment that would start in 2010. GERDA-I will scrutinize the KHDK claim and reach a sensitivity of $\mathrm{T}_{1 / 2}>2 \times 10^{25} \mathrm{y}(90 \% \mathrm{CL})$ after two years of data taking. $40 \mathrm{~kg}$ of germanium isotopically enriched in ${ }^{76} \mathrm{Ge}$ are already available for GERDA-II phase.

CUORE citeqprop05 will consist of an array of $988 \mathrm{TeO}_{2}$ bolometers arranged in a cylindrical configuration of 19 towers containing 52 crystals each $(750 \mathrm{~g}$ ), for a total mass of $\sim 741 \mathrm{~kg}$. Each of these towers is a CUORICINO-like detector consisting of 13 modules, 4 detectors each. The expected energy resolution is $\sim 5 \mathrm{keV}$ FWHM at the $0 v \beta \beta$ transition energy $(\sim 2.53 \mathrm{MeV})$. A background level of of the order of $\sim 0.01 \mathrm{c} / \mathrm{keV} / \mathrm{kg} / \mathrm{y}$ is expected by extrapolating the CUORICINO background results and the dedicated CUORE R\&D measurements. The expected 5y sensitivity is $2.1 \times 10^{26} \mathrm{y}$. CUORE will therefore allow a closer look at the IH region of neutrino masses. CUORE is presently under construction at LNGS at a relatively low cost thanks to the high natural abundance of ${ }^{130} \mathrm{Te}$. Setup completion is expected in 2013. Thanks to the bolometer's versatility, alternative options with respect to $\mathrm{TeO}_{2}$ are also possible. In particular, promising results have been recently obtained with scintillating bolometers [26] which could allow to study in the future new $0 v \beta \beta$ active isotopes with improved sensitivity.

EXO [27] is LXe TPC detector with an improved energy resolution of 3.3\% FWHM thanks to the simultaneous measurement of ionization and scintillation light. A smaller prototype experiment with a mass of $200 \mathrm{~kg}\left(80 \%{ }^{136} \mathrm{Xe}\right)$, is presently being installed at WIPP. The primary goal is to measure the ${ }^{136} \mathrm{Xe} 2 v \beta \beta$ half-life and to study the $0 v \beta \beta$ decay with a sensitivity of $\sim 10^{25} \mathrm{y}$ in two years of data taking. The full scale experiment foresees $\sim 1-10$ tons of isotopically enriched $\left(85 \%\right.$ in $\left.{ }^{136} \mathrm{Xe}\right) \mathrm{Xe}$ detector with a tagging of the doubly charged Ba isotope produced in the decay ${ }^{136} \mathrm{Xe} \rightarrow{ }^{136} \mathrm{Ba}^{++}+2 e^{-}$. The tagging would allow a complete background suppression although the technical feasibility of such an ambitious project requires a hard and still ongoing R\&D phase. The unavoidable $2 v \beta \beta$ contribution is a serious concern due to the poor energy resolution of Xe detectors.

Both SNO+ and KAMLAND experiments use a different strategy: the $0 v \beta \beta$ active isotope is dispersed in a large mass of low-radioactive scintillator. They aim to compensate the modest energy resolution with a large statistics. SNO+ is pursuing the goal of studying ${ }^{150} \mathrm{Nd}$ with 50 $500 \mathrm{~kg}$ of isotopically enriched Neodimium loaded liquid scintillator depending on the results of the currently ongoing R\&D program. With a energy resolution of $\sim 6 \%$ FWHM a 3 y sensitivity of $\sim 4 \times 10^{26}$ y could be reached. This search will depend critically on the possibility to enrich $\mathrm{Nd}$ in ${ }^{150} \mathrm{Nd}$. A similar approach is proposed by KAMLAND but for the ${ }^{136} \mathrm{Xe}$ active isotope. Their program should start in 2011 (first phase) with 200-400 kg of isotope and continue in 2013 with 1 ton of Xenon enriched to $90 \%$ in ${ }^{136} \mathrm{Xe}$. A preliminary estimate of the $5 \mathrm{y}$ sensitivity for phase I amounts to $\sim 10^{26} \mathrm{y}$.

The only experiment based on an inhomogeneous technique is Super-NEMO. It is an extension of the successful NEMO3 concept, properly scaled in order to accommodate $\sim 100 \mathrm{~kg}$ of ${ }^{82} \mathrm{Se}$ foils spread among 20 detector modules. The proposed geometry is planar. The energy resolution will be improved from $12 \%$ FWHM to $7 \%$ FWHM to improve the signal detection efficiency from $8 \%$ to $40 \%$ and reduce the $2 v \beta \beta$ contribution. The detector modules will have an active water shield to further reduce cosmic ray backgrounds. The proposed detector dimensions will require a larger hall than is currently available at Frejus and an expansion of the facility is therefore required and 
actively pursued. A demonstrator (single module) is expected to be completed in 2011 and operated in the current NEMO3 site.

\section{Conclusions}

The existence of a non-vanishing neutrino mass, provided by neutrino oscillation experiments, has definitively renewed the interest in Neutrinoless Double Beta Decay as the only search able to test the Majorana neutrino nature. Its observation, will prove unambiguously the Majorana nature of the neutrino mass thus implying new Physics beyond the Standard Model. Moreover this process allows to investigate the neutrino absolute mass scale through the measurement of the effective Majorana mass of the electron neutrino.

Present $m_{\beta \beta}$ sensitivities are still outside the range required to test the inverted neutrino mass hierarchy. However an extensive program of new $0 v \beta \beta$ experimenst is supported to pursue such a goal. The success of the program strongly depends on the true capability of the proposed projects to reach the required background levels in the $0 v \beta \beta$ region.

The claimed evidence for a $0 v \beta \beta$ signal in the HM data will be verified soon by the forthcoming next generation experiments.

In particular, if neutrinos are Majorana fermions, next generation experiments will be able to solve the problem of the neutrino mass hierarchies.

\section{References}

[1] S. Fukuda et al., Phys. Lett. 81, 2644, 1999

[2] Q.R. Ahmad et al., Phys. Rev. Lett. 87, 071301, 2001 Phys. Rev. Lett. 89, 011301, 2002, Phys. Rev. Lett. 89, 011302, 2002

[3] S.N. Ahmad et al., Phys. Rev. Lett. 92, 181301, 2004

[4] K. Eguchi et al., Phys. Rev. Lett. 90, 021802, 2003

[5] M. H. Ahn et al., Phys. Rev. Lett. 90, 041801, 2003

[6] E. Majorana, Nuovo Cim. 14, 171, 1937

[7] M. Goeppert-Mayer, Phys. Rev. 48, 512, 1935

[8] W.H. Furry, Phys. Rev. 56, 1184, 1939

[9] A. Strumia and F. Vissani, hep-ph/0606054

[10] S. Pascoli, S.T. Petcov and T. Schwetz, hep-ph/0505226

[11] H.V. Klapdor-Kleingrothaus et al. (HM Coll.), Eur. Phys. J. A 12, 147, 2001 and hep-ph/0103062

[12] C.E. Aalseth et al., Phys. Rev. D 65, 092007, 2002

[13] A.S. Barabash, hep-ex/0610025

[14] K. Lang (NEMO3 coll.), Proc. fo the WIN09 conference, Perugia September 2009, Italy

[15] C. Arnaboldi et al., Phys. Rev. C 78, 035502, 2008

[16] H.V. Klapdor-Kleingrothaus, Phys. Scr. T 127, 40, 2006 H.V. Klapdor-Kleingrothaus and I.V. Krivosheina, Modern Physics Letters A 21, 1547, 2006 
[17] A.S. Barabash, nucl-ex/0908.4173

[18] I. Ogawa et al., Nucl. Phys. A 730, 215, 2004

[19] F.A. Danevich et al., Phys. Rev. C 68, 03550, 2003

[20] J.M. Gavriljuk et al., Phys. Rev. C 61, 035501, 2000

[21] R. Bernabei, Phys. Lett. B 546, 23, 2002

[22] A.S. Barabash, Phys. At. Nucl. 68, 414, 2005

[23] I. Abt et al., hep-ex/0404039

[24] H.V. Klapdor-Kleingrothaus HV, hep-ph/0103074

[25] C. Arnaboldi et al., hep-ex/0501010

[26] S.Pirro et al., Phys. Atom. Nucl. 69, 2109, 2006

[27] M. Danilov et al., Phys. Lett. B 480, 12, 2000 